PROCEEDINGS OF THE

AMERICAN MATHEMATICAL SOCIETY

Volume 135, Number 11, November 2007, Pages 3547-3552

S 0002-9939(07)08859-4

Article electronically published on July 3, 2007

\title{
ON THE ALGEBRAIC CLOSURE IN RINGS
}

\author{
DRAGANA CVETKOVIC-ILIC AND ROBIN HARTE
}

(Communicated by Joseph A. Ball)

\begin{abstract}
The "algebraic closure" of a subset $K \subseteq A$ of a ring is an algebraic analogue of topological closure.
\end{abstract}

Suppose $A$ is a ring, or additive category, with identity 1 and invertible group $A^{-1}$. Then we make the following

Definition 1. The algebraic closure of a subset $K \subseteq A$ is the set

$$
\operatorname{cl}_{\mathrm{alg}}(K) \equiv \mathrm{cl}_{\mathrm{alg}}^{A}(K)=\left\{a \in A: \forall b, c \in A \exists a^{\prime} \in K, 1-b\left(a-a^{\prime}\right) c \in A^{-1}\right\} .
$$

Equivalently

$$
a \in \operatorname{cl}_{\text {alg }}(K) \Longleftrightarrow \forall b \in A \exists a^{\prime} \in K, 1-b\left(a-a^{\prime}\right) \in A^{-1}
$$

for if this holds, then for arbitrary $b, c \in A$ there is $a^{\prime} \in A$ with

$$
1-c b\left(a-a^{\prime}\right) \in A^{-1} \Longleftrightarrow 1-b\left(a-a^{\prime}\right) c \in A^{-1} .
$$

For example if $A$ is a Banach algebra, then the norm closure is a subset of the algebraic closure: using the geometric series [10.

$$
\|z\|\left\|a-a^{\prime}\right\|\|w\|<1 \Longrightarrow 1-z\left(a-a^{\prime}\right) w \in A^{-1} .
$$

The reverse inclusion is not clear: for example

$$
\operatorname{cl}_{\mathrm{alg}}\{a\}=a+\operatorname{Rad}(A),
$$

the algebraic closure of a singleton is the coset modulo the Jacobson radical.

The algebraic closure has almost all of the properties of topological closure, and is compatible with the algebraic operations:

Theorem 2. For arbitrary $K, H \subseteq A$ there is inclusion

$$
K \subseteq \operatorname{cl}_{\text {alg }}(K),
$$

implication

$$
K \subseteq H \Longrightarrow \operatorname{cl}_{\text {alg }}(K) \subseteq \operatorname{cl}_{\text {alg }}(H),
$$

and inclusion (necessarily equality)

$$
\operatorname{cl}_{\text {alg }} \operatorname{cl}_{\text {alg }}(K) \subseteq \operatorname{cl}_{\text {alg }}(K) .
$$

Received by the editors September 28, 2005 and, in revised form, July 10, 2006.

2000 Mathematics Subject Classification. Primary 46L05.

Key words and phrases. ring, algebraic closure, invertibility.

The first author was supported by Grant no. 144003 of Ministry of Science, Republic of Serbia.

(C)2007 American Mathematical Society Reverts to public domain 28 years from publication 
There is also inclusion

$$
\operatorname{cl}_{\mathrm{alg}}(K)+\operatorname{cl}_{\mathrm{alg}}(H) \subseteq \operatorname{cl}_{\mathrm{alg}}(K+H)
$$

and

$$
\operatorname{cl}_{\mathrm{alg}}(K) \cdot \mathrm{cl}_{\mathrm{alg}}(H) \subseteq \mathrm{cl}_{\mathrm{alg}}(K \cdot H) .
$$

Proof. (2.1) and (2.2) are clear; towards (2.3), if $x \in \mathrm{cl}_{\mathrm{alg}} \mathrm{cl}_{\mathrm{alg}}(K)$, then for arbitrary $z, w \in A$ there is $x^{\prime} \in \mathrm{cl}_{\text {alg }}(K)$ for which $1-z\left(x-x^{\prime}\right) w=c \in A^{-1}$, and then $x^{\prime \prime} \in K$ for which $1-c^{-1} z\left(x^{\prime}-x^{\prime \prime}\right) w \in A^{-1}$. Now

$$
1-z\left(x-x^{\prime \prime}\right) w=1-z\left(x-x^{\prime}\right) w-z\left(x^{\prime}-x^{\prime \prime}\right) w=c\left(1-c^{-1} z\left(x^{\prime}-x^{\prime \prime}\right) w\right) \in A^{-1} \text {. }
$$

Towards (2.4) and (2.5), if $x \in \operatorname{cl}_{\text {alg }}(K)$ and $y \in \operatorname{cl}_{\text {alg }}(H)$ and if $z, w \in A$, then, with $1-z\left(x-x^{\prime}\right) w=c \in A^{-1}$,

$$
\begin{aligned}
1-z\left(x+y-x^{\prime}-y^{\prime}\right) w & =1-z\left(x-x^{\prime}\right) w-z\left(y-y^{\prime}\right) w \\
& =\left(1-z\left(x-x^{\prime}\right) w\right)\left(1-c^{-1} z\left(y-y^{\prime}\right) w\right) \in A^{-1} A^{-1}=A^{-1}
\end{aligned}
$$

provided $1-c^{-1} z\left(y-y^{\prime}\right) w \in A^{-1}$ and, with $1-z\left(x-x^{\prime}\right) y w=d \in A^{-1}$,

$$
\begin{aligned}
1-z\left(x y-x^{\prime} y^{\prime}\right) w & =1-z\left(x-x^{\prime}\right) y w-z x^{\prime}\left(y-y^{\prime}\right) w \\
& =\left(1-z\left(x-x^{\prime}\right) y w\right)\left(1-d^{-1} z x^{\prime}\left(y-y^{\prime}\right) w\right) \in A^{-1}
\end{aligned}
$$

provided $1-d^{-1} z x^{\prime}\left(y-y^{\prime}\right) w \in A^{-1}$.

One of the Kuratowski closure axioms however seems to fail:

Example 3. For $K, H \subseteq A$, inclusion

$$
\operatorname{cl}_{\mathrm{alg}}\left(K_{\cup} H\right) \subseteq \mathrm{cl}_{\mathrm{alg}}(K) \cup \mathrm{cl}_{\mathrm{alg}}(H)
$$

is liable to fail.

Proof. In the ring $A=\mathbf{C}^{2}$ take

$$
K=\{1\} \times \mathbf{C}, H=\{-1\} \times \mathbf{C}
$$

and claim

$$
(2,3) \in \operatorname{cl}_{\mathrm{alg}}\left(K_{\cup} H\right) \backslash\left(\operatorname{cl}_{\mathrm{alg}} K_{\cup} \mathrm{cl}_{\mathrm{alg}} H\right) .
$$

To see that $(2,3) \notin \mathrm{cl}_{\text {alg }} K$ notice

$$
(1,1)-(1,1)((2,3)-(1, t))=(0, t-2) \notin A^{-1} .
$$

To see that $(2,3) \notin \operatorname{cl}_{\text {alg }} H$ notice

$$
(1,1)-(1 / 3,1)((2,3)-(-1, t))=(0, t-2) \notin A^{-1} .
$$

Finally to see that $(2,3) \in \operatorname{cl}_{\mathrm{alg}}\left(K_{\cup} H\right)$ suppose $(r, s) \in A$ is arbitrary. If $r \neq 1$, then for arbitrary $t \in \mathbf{C}$

$$
(1,1)-(r, s)((2,3)-(1, t))=(1-r, 1-s(t-3)) \in A^{-1} \text { if } 1-s(3-t) \neq 0 .
$$

If $r=1$, then for arbitrary $t \in \mathbf{C}$

$$
(1,1)-(r, s)((2,3)-(-1, t))=(-2,1-s(3-t)) \in A^{-1} \text { if } 1-s(3-t) \neq 0 .
$$

The algebraic closure of the invertibles participates in a curious minuet with the left and the right invertibles: 
Theorem 4. There is equality

$$
A_{\text {left }}^{-1} \cap \mathrm{cl}_{\mathrm{alg}} A^{-1}=A^{-1}=A_{\mathrm{right}}^{-1} \cap \mathrm{cl}_{\mathrm{alg}} A^{-1},
$$

and inclusion

$$
\operatorname{cl}_{\text {alg }} A^{-1} \subseteq A^{-1}+A^{-1}+A^{-1} .
$$

Necessary and sufficient for

$$
0 \in \operatorname{cl}_{\text {alg }} A^{-1}
$$

is inclusion

$$
A \subseteq A^{-1}+A^{-1} .
$$

Proof. Towards (4.1) we prove more, and claim

$$
A_{\text {left } \cap}^{-1} \mathrm{cl}_{\mathrm{alg}} A_{\text {right }}^{-1}=A^{-1}=A_{\text {right }}^{-1} \cap \mathrm{cl}_{\mathrm{alg}} A_{\text {left }}^{-1} .
$$

Towards the first equality suppose $a \in A_{\text {left } \cap \text { cl }}^{-1} \operatorname{lalg}_{\text {right }}^{-1}$ so that there are $a^{\prime}, b, b^{\prime} \in A$ for which $a^{\prime} a=1=b b^{\prime}$ with $1-a^{\prime}(a-b) \in A^{-1}$. But this means $a^{\prime} a=1$ with $a^{\prime} b \in A^{-1}$; hence, $b \in A_{\text {left }}^{-1}$ and so $b \in A^{-1}$. The argument for the second equality in (4.5) is the same.

Towards (4.2) suppose $a \in \mathrm{cl}_{\text {alg }} A^{-1}$. Then for arbitrary $b \in A^{-1}$ there is $c \in A^{-1}$ for which

$$
1-b(a-c)=d \in A^{-1} \Longrightarrow a=b^{-1}(1-d)+c \in A^{-1}+A^{-1}+A^{-1} .
$$

Finally if (4.3) holds, then for arbitrary $b \in A$ there is $c \in A^{-1}$ for which $1-b(-c)=$ $d \in A^{-1}$, giving $b=(d-1) c^{-1} \in A^{-1}+A^{-1}$, as in (4.4). Conversely for arbitrary $c, d \in A^{-1}$ we have

$$
1-(c+d) d^{-1}=-c d^{-1} \in A^{-1} ;
$$

in the presence of (4.4) this is (4.3)

The analogue of (4.1) for the topological closure is familiar in Banach algebras [8], 10. From (4.1) it follows that everything in the algebraic closure of the invertibles is "consistent in regularity" [6], 7]:

$$
\begin{aligned}
\operatorname{cl}_{\text {alg }} A^{-1} & \subseteq A^{-1} \cup\left(A \backslash\left(A_{\text {left }}^{-1} A_{\text {right }}^{-1}\right)\right) \\
& =\left\{a \in A: a x \in A^{-1} \Longleftrightarrow x a \in A^{-1}\right\} .
\end{aligned}
$$

The last part of Theorem 4 generalizes to radical elements and to idempotents:

Theorem 5. If

$$
1+q A^{-1} \subseteq A^{-1},
$$

then the condition (4.3) is necessary and sufficient for

$$
q \in \operatorname{cl}_{\mathrm{alg}} A^{-1} .
$$

If instead $p=p^{2} \in A$ is idempotent, then

$$
0 \in \mathrm{cl}_{\text {alg }}^{A}(p A p)^{-1} \Longleftrightarrow 0 \in \operatorname{cl}_{\text {alg }}^{p A p}(p A p)^{-1} \Longrightarrow 1-p \in \operatorname{cl}_{\text {alg }} A^{-1} .
$$


Proof. If $q \in A$ is in $\operatorname{cl}_{\text {alg }} A^{-1}$, then for arbitrary $b \in A$ there is $c \in A^{-1}$ for which $1-b(q-c)=d \in A^{-1}$ and hence

$$
\text { be }=d c^{-1}-c^{-1} \text { with } e=1-q c^{-1} .
$$

Now if (5.1) holds, then $e \in A^{-1}$ giving $b=(d-1)(e c)^{-1} \in A^{-1}+A^{-1}$, which is (4.3). Conversely for arbitrary $c, d \in A^{-1}$

$$
1-(c+d)\left(q+c^{-1}\right)=-\left(d c^{-1}+c q+d q\right) \in A^{-1}+A^{-1} q+A^{-1} q,
$$

which is included in $A^{-1}$ by (5.1). In the presence of (4.3) this applies to arbitrary $b=c+d \in A$. Towards (5.3), if 0 is the algebraic closure in $p A p$ of the invertible group $(p A p)^{-1}$, then for arbitrary $b \in A$ there is $c=p c p \in(p A p)^{-1}$ for which $p(1+b c) \in(p A p)^{-1}$, giving

$$
1+p b c=1-p+p(1+b c) \in A^{-1},
$$

which says $0 \in \operatorname{cl}_{\text {alg }}^{A}(p A p)^{-1}$. Conversely if this holds, then for arbitrary $b \in A$, in particular $p b p \in p A p$, there is $c=c p c \in(p A p)^{-1}$ and $d \in A$ for which $(1+p b c) d=$ $1=d(1+p b c)$, giving

$$
(p+p b c) p d p=p=p d(p+p b c)
$$

and hence $p(1+b c) \in(p A p)^{-1}$. Also if for arbitrary $b \in A$ there is $c, d \in p A p$ with $1-b c \in A^{-1}$ and $c d=p=d c$, then $a=1-p-c \in A^{-1}$ with $a^{-1}=1-p-d$ giving $1-b a=1-b c \in A^{-1}$.

The algebraic closure intervenes in generalized inverse theory:

Lemma 6. With

$$
\hat{A}=\{a \in A: a \in a A a\} \text { and } \check{A}=\left\{a \in A: a \in a A^{-1} a\right\}
$$

there is inclusion

$$
\hat{A}_{\cap} \mathrm{cl}_{\mathrm{alg}} A^{-1} \subseteq \check{A} .
$$

Necessary and sufficient for equality in (6.2) is that

$$
A^{\bullet} \equiv\left\{p \in A: p=p^{2}\right\} \subseteq \mathrm{cl}_{\mathrm{alg}} A^{-1} .
$$

Proof. If $a=a a^{\prime} a \in \hat{A}$, so that $a^{\prime} a=p=p^{2}$ is idempotent, and if

$$
b \in A^{-1} \text { with } 1+(b-a) a^{\prime}=c^{-1} \in A^{-1},
$$

then

$$
a=(c b) p \in \check{A} .
$$

For equality in (6.2), observe [9], 10

$$
\check{A}=A^{-1} A^{\bullet}=A^{\bullet} A^{-1} \text {. }
$$

In Banach algebras (6.3) is clear [9], 10]: generally

$$
0 \notin \operatorname{int} \sigma(a) \Longrightarrow a \in \operatorname{cl}_{\mathrm{alg}} A^{-1} \text {, }
$$

where $\sigma(a)=\left\{\lambda \in \mathbf{C}: a-\lambda \notin A^{-1}\right\}$ is the usual spectrum.

We can extend the algebraic closure to tuples:

Definition 7. For arbitrary $K \subseteq A^{n}$

$$
\operatorname{cl}_{\mathrm{alg}} K=\left\{x \in A^{n}: \forall z, w \in A^{n} \exists x^{\prime} \in K, 1-\sum_{j=1}^{n} z_{j}\left(x_{j}-x_{j}^{\prime}\right) w_{j} \in A^{-1}\right\} .
$$


We can also define left and right invertible tuples:

$$
A_{\text {left }}^{-n}=\left\{a \in A^{n}: 1 \in \sum_{j=1}^{n} A a_{j}\right\} \text { and } A_{\text {right }}^{-n}=\left\{a \in A^{n}: 1 \in \sum_{j=1}^{n} a_{j} A\right\} .
$$

If we interpret $A$ as an additive category, then in a sense we have already dealt with $n$-tuples, and more generally $n \times m$ matrices, over $A$, which just form another additive category $B=\operatorname{Matrix}(A)$; thus most of Theorem 2 extends to $n$-tuples. For example the analogues of (2.1) and (2.2) for $K, H \subseteq A^{n}$ are immediate, while for the analogue of (2.3) we argue that if $x \in \mathrm{cl}_{\text {alg }} \mathrm{cl}_{\text {alg }}(K)$, then for arbitrary $z, w \in A^{n}$ there is $x^{\prime} \in \operatorname{cl}_{\mathrm{alg}}(K)$ for which $1-\sum_{j} z_{j}\left(x_{j}-x_{j}^{\prime}\right) w_{j}=c \in A^{-1}$, and then $x^{\prime \prime} \in K$ for which $1-c^{-1} \sum_{j} z_{j}\left(x_{j}^{\prime}-x_{j}^{\prime \prime}\right) w_{j} \in A^{-1}$. Now

$$
\begin{aligned}
1-\sum_{j} z_{j}\left(x_{j}-x_{j}^{\prime \prime}\right) w & =1-\sum_{j}\left(z_{j}\left(x_{j}-x_{j}^{\prime}\right) w_{j}-z_{j}\left(x_{j}^{\prime}-x_{j}^{\prime \prime}\right) w_{j}\right) \\
& =c\left(1-c^{-1} \sum_{j} z_{j}\left(x_{j}^{\prime}-x_{j}^{\prime \prime}\right) w_{j}\right) \in A^{-1} .
\end{aligned}
$$

Towards the extension of (2.4), if $x \in \mathrm{cl}_{\mathrm{alg}}(K)$ and $y \in \mathrm{cl}_{\mathrm{alg}}(H)$ and if $z, w \in A^{n}$ then, with $1-\sum_{j} z_{j}\left(x_{j}-x_{j}^{\prime}\right) w_{j}=c \in A^{-1}$,

$$
\begin{aligned}
1- & \sum_{j} z_{j}\left(x_{j}+y_{j}-x_{j}^{\prime}-y_{j}^{\prime}\right) w_{j} \\
& =\left(\sum_{j}\left(1-z_{j}\left(x_{j}-x_{j}^{\prime}\right) w_{j}\right)\left(1-c^{-1} \sum_{j} z_{j}\left(y_{j}-y_{j}^{\prime}\right) w_{j}\right)\right. \\
& \in A^{-1} A^{-1}=A^{-1} \text { provided } 1-c^{-1} \sum_{j} z_{j}\left(y_{j}-y_{j}^{\prime}\right) w_{j} \in A^{-1} .
\end{aligned}
$$

Similarly, for an extended version of (2.5), with $1-\sum_{j} z_{j}\left(x_{j}-x_{j}^{\prime}\right) y_{j} w_{j}=d \in$ $A^{-1}$

$$
\begin{aligned}
1- & \sum_{j} z_{j}\left(x_{j} y_{j}-x_{j}^{\prime} y_{j}^{\prime}\right) w_{j} \\
& =\left(1-\sum_{j} z_{j}\left(x_{j}-x_{j}^{\prime}\right) y_{j} w_{j}\left(1-d^{-1} \sum_{j} z_{j} x_{j}^{\prime}\left(y_{j}-y_{j}^{\prime}\right) w_{j}\right)\right. \\
& \in A^{-1} \text { provided } 1-d^{-1} \sum_{j} z_{j} x_{j}^{\prime}\left(y_{j}-y_{j}^{\prime}\right) w_{j} \in A^{-1} .
\end{aligned}
$$

(2.5) would also extend, in a category $A$, to $K, H$ and a more general bilinear image $K * H$.

Declare that a ring $A$ has left stable range $\leq n$ provided

$$
\forall(a, b) \in A^{n} \times A,(a, b) \in A_{\mathrm{left}}^{-n-1} \Longrightarrow \exists c \in A^{n}, a-c b \in A_{\mathrm{left}}^{-n} .
$$

Corach and Suarez [4], 5], and Blackadar [3], have considered this kind of situation when $A$ is commutative, or a $C^{*}$-algebra.

Notice how the definition prefers the final element of an $n+1$ tuple; an alternative would say that if $d \in A^{n+1}$ was "left invertible", then there would exist an index $1 \leq j \leq n+1$ for which an analogue of (7.3) held. In commutative Banach algebras the condition that $A$ has stable range $\leq 1$ reduces to the topological closure of the invertible group being the whole of $A$. We offer here a curious hybrid result: 
Theorem 8. If

$$
A \subseteq \operatorname{cl}_{\text {alg }} A_{\text {left }}^{-1},
$$

then there is implication

$$
(a, b) \in A_{\text {left }}^{-2} \Longrightarrow(a-A b)_{\cap} A_{\text {right }}^{-1} \neq \emptyset .
$$

Proof. Suppose

$$
a^{\prime} a+b^{\prime} b=1 \text { with } a^{\prime} \in \operatorname{cl}_{\text {alg }} A_{\text {left }}^{-1},
$$

so that there are $a^{\prime \prime}, a^{\prime \prime \prime}$ in $A$ for which

$$
b^{\prime} b=1-a^{\prime} a=d-a^{\prime \prime} a \text { with } d \in A^{-1} \text { and } a^{\prime \prime \prime} a^{\prime \prime}=1
$$

giving

$$
a-c b=a^{\prime \prime \prime} d \in A_{\text {right }}^{-1} \text { with } c=-a^{\prime \prime \prime} b^{\prime} .
$$

When $b=0$ this gives back (4.1). The same argument gives back a weaker version of (7.3): if $(a, b) \in A \times A^{n}$, then if (8.1) holds there is implication

$$
(a, b) \in A_{\text {left }}^{-n-1} \Longrightarrow\left(a-\sum_{j} A b_{j}\right)_{\cap} A_{\text {right }}^{-1} \neq \emptyset .
$$

We remark that the condition (8.2) says that the element $a \in A$ is in another kind [1, 2] of "closure" of the semigroup $A_{\text {right }}^{-1}$ : for the moment we find the precise relationship between this condition and ours elusive.

\section{REFERENCES}

1. P. Ara, G.K. Pedersen and F. Perera, An infinite analogue of rings with stable rank one, J. Algebra 230 (2000) 608-655. MR 1775806 (2001g:16018)

2. P. Ara, G.K. Pedersen and F. Perera, A closure operation in rings, Int. J. Math. 12 (2001) 791-812. MR1850672 (2002i:16010)

3. B. Blackadar, The stable rank of full corners in $C^{*}$-algebras, Proc. Amer. Math. Soc. 132 (2004) 2945-2950. MR2063114 (2005d:46111)

4. G. Corach and F.D. Suarez, Extension problems and stable rank in commutative Banach algebras, Topology and Its Appl. 21 (1985) 1-8. MR0808718 (87a:46086)

5. G. Corach and F.D. Suarez, Thin spectra and stable range, J. Funct. Anal. 81 (1988) 432-442. MR 0971887 (89k:46061)

6. D. Djordjevic, Operators consistent in regularity, Publ. Math. (Debrecen) 60 (2002) 1-15. MR:1990872 (2004m:47001)

7. D. Djordjevic, R.E. Harte and C. Stack, On left-right consistency in rings, Math. Proc. Royal Irish Acad. 106A (1) (2006) 11-17. MR 2198248 (2006i:46066)

8. R.E. Harte, Almost open mappings between normed spaces, Proc. Amer. Math. Soc. 90 (1984) 243-249. MR0727242 (85b:47024)

9. R.E. Harte, Regular boundary elements, Proc. Amer. Math. Soc. 99 (1987) 328-330. MR0870795 (88d:46088)

10. R.E. Harte, Invertibility and singularity for bounded linear operator, Dekker 1988. MR0920812 (89d:47001)

Department of Mathematics, Faculty of Science and Mathematics, University of Nis, Visegradska 33, 18000 Nis, SERBia

E-mail address: gagamaka@ptt.yu

School of Mathematics, Trinity College, Dublin, Ireland

E-mail address: rharte@maths.tcd.ie 